\title{
USP1 keeps ID proteins stable
}

Basic helix-loop-helix transcription factors, which are regulators of development and differentiation, are inhibited by the DNA-binding protein inhibitors ID1, ID2, ID3 and ID4. ID proteins are abundant in embryonic and adult stem cells, and are overexpressed in some dedifferentiated primary tumours, suggesting that they might help to maintain stem cell fate. ID1, ID2 and ID3 are subject to Lys48-linked ubiquitylation followed by degradation, a regulatory mechanism that might be aberrant in tumours. Williams et al. now identify USP1 as a deubiquitylating enzyme (DUB) for ID1 to ID3 that stabilizes these proteins to promote stem cell characteristics in both osteosarcoma and mesenchymal stem cells (MSCs).

The authors first overexpressed 94 human DUBs in cells and monitored ID2 protein levels. Only USP1 increased the endogenous levels of ID2 and interacted with, and had deubiquitylating activity against, this protein. Furthermore, USP1, but not a catalytically inactive USP1, reduced ID2 ubiquitylation in vitro and in vivo. Thus, USP1 is a DUB for ID2 and, as indicated by a subset of the above experiments, also for ID1 and ID3.

On carrying out microarray analysis of healthy and diseased tissue, the authors observed that USP1 mRNA and protein levels were increased in a subset of primary human osteosarcoma biopsy samples, and that this correlated with increased ID2 protein levels. USP1 and ID2 levels were also elevated in human osteosarcoma cell lines and primary osteoblasts; short hairpin RNA (shRNA)-mediated knockdown of USP1 caused a reduction in levels of ID1, ID2 and ID3 in these cells. So, USP1 stabilizes ID proteins in osteosarcoma.

Osteosarcomas, which are likely to develop from MSCs, consist of all three MSC lineages (that is, osteoblasts, chondrocytes and adipocytes) and express MSC markers rather than osteoblast markers. As ID proteins have been implicated in stem cell maintenance, the authors sought to determine whether their depletion promoted osteoblast differentiation. Indeed, expression of USP1 or ID shRNAs in osteosarcoma cells decreased and increased, respectively, the expression of MSC and osteoblast markers. Thus, osteogenic differentiation in osteosarcomas is blocked by a USP1-mediated increase in ID proteins. Furthermore, USP1 overexpression in normal human MSCs increased ID protein levels and cell proliferation, and reduced the expression of osteoblast markers. Therefore, an increase in USP1 levels, and thus ID levels, also prevents the differentiation of normal MSCs, thereby favouring stem cell maintenance.

Finally, the authors examined the effect of altered USP1 expression in animal models of tumorigenesis and of normal skeletal development. The implantation of cells overexpressing USP1 or ID2 into mice resulted in the development of tumours; in vitro assays confirmed that IDs are essential for the ability of USP1 to transform cells. In assessing bone development, the authors concluded that USP1-null mice had skeletal defects that are consistent with the effects of USP1 expression levels in MSCs.

So, this study identifies USP1 as a DUB for ID1 to ID3, and shows that it stabilizes these proteins to inhibit the differentiation of MSCs in both normal stem cells and osteosarcoma.

Katharine H. Wrighton

ORIGINAL RESEARCH PAPER Williams, S. A. et al. USP1
deubiquitinates ID proteins to preserve a mesenchymal stem cell
program in osteosarcoma. Cell 146, 918-930 (2011)
FURTHER READING Komander, K., Clague, M. J. \& Urbé, S. Breaking
the chains: structure and function of the deubiquitinases. Nature
Rev. Mol. Cell Biol. 10, 550-563 (2009)

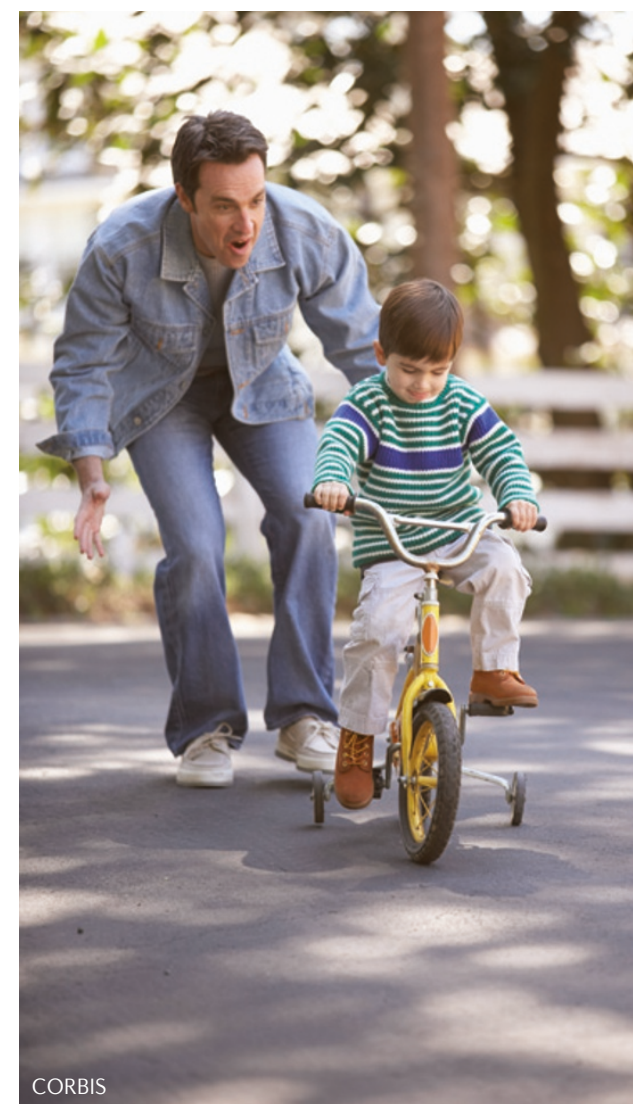

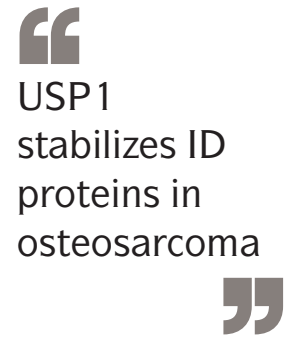

stabilizes ID

proteins in

osteosarcoma 